Article

\title{
Internal Model Control for Rank-Deficient System with Time Delays Based on Damped Pseudo-Inverse
}

\author{
Meiying Jiang ${ }^{1}$, Beiyan Jiang ${ }^{2,3, *}$ and Qi Wang ${ }^{1}$ \\ 1 School of Information Science and Technology, Beijing University of Chemical Technology, No. 15, \\ Beisanhuan East Road, Beijing 100029, China; jmy@mail.buct.edu.cn (M.J.); wangq@mail.buct.edu.cn (Q.W.) \\ 2 Beijing Key Laboratory of Information Service Engineering, Beijing Union University, \\ No. 97 Beisihuan East Road, Chao Yang District, Beijing 100101, China \\ 3 College of Robotics, Beijing Union University, No. 4 Gongti North Road, Chaoyang District, \\ Beijing 100027, China \\ * Correspondence: jqrbeiyan@buu.edu.cn
}

Received: 9 March 2019; Accepted: 29 April 2019; Published: 6 May 2019

\begin{abstract}
It is a challenge to design a satisfactory controller for a complex multivariable industrial system with minimal offsetting and a slow response. An internal model control method is proposed for rank-deficient systems with a time delay based on a damped pseudo-inverse. An internal model control was designed to obtain the desired dynamic characteristics of the system by transforming the time-delay system into a system without a time delay, following the Pade approximation approach. By introducing a damping factor, the internal model controller was designed based on a damped pseudo-inverse, since the inverse matrix of the rank-deficient system does not exist. Furthermore, a singular value decomposition was used to analyze the steady-state performance of the system. The selection of the damping factor was also presented, and a $\mu$ analysis was made to evaluate the stability of the system. To demonstrate the effectiveness of the proposed method, a crude distillation process with five inputs and four outputs was considered as an example. The simulation results illustrate that not only can the proposed strategy guarantee the system's stability, but it also has a relatively good dynamic performance.
\end{abstract}

Keywords: internal model control; rank-deficient system; time delay; damped pseudo-inverse

\section{Introduction}

Along with the rapid development of the industrial process, the controlled object models are becoming more and more complex. Multivariable systems are widely exhibited in most actual industrial processes. The problem is usually further complicated by the multivariable nature of most plants, leading to offsetting and a slow response. Designing a satisfactory controller for a multivariable system is more difficult than for a single-variable one [1,2]. Therefore, it is a challenge to design and implement a controller to impose a desired behavior and performance on these multivariable systems. There are plenty of ways to design controllers, such as robust control [3-5], intelligent control [6,7], and internal model control (IMC) [8-10]. As one of the advanced control arithmetics in process control, IMC has been shown to be a powerful method for the control of multivariable systems due to its good tracking performance and the robustness properties of the IMC structure, in which the controller implementation includes an explicit plant model in parallel with the plant [11]. Therefore, it shows a strong advantage in the theoretical analysis of the control system stability and robustness, especially in the control of large time-delay systems, where the effect is particularly significant. Due to its formation, it has been not only frequently applied in the process control with a slow response, but it has also acquired a more superior effect than proportion integration differentiation (PID) in the motor control with a fast response. 
The complex industrial production process always needs to use multiple controlled variables and multiple manipulated variables to describe a controlled system. Processes with an equal number of inputs and outputs are known as square systems, while those with unequal numbers are known as non-square systems. Over the past few decades, IMC was used to study the control problem for both square systems and non-systems. For example, for the multivariable plants that are subject to multiple operating regimes [12], a new method was proposed to reduce the inherent conservatism in the existing design methods by incorporating the global multiple-model into a decentralized IMC. Tang et al. [13] compensated the system's time delay and improved the system's stability by constructing a plant matrix for the dual-input and dual-output networked control system in an online, real-time and dynamic manner. Garridoa et al. [14] optimized the internal model control main controller for the multi-time-delay stable multi-variable process by adding the inverse decoupling structure of a diagonal filter. By introducing the concept of the generalized inverse, Yao et al. studied the IMC for the non-square system [11]. Chen et al. extended the method to a non-square system with multiple time delays and right-half-plant zeros when parameters uncertainty exists [15]. But it is difficult to calculate the pseudo-inverse of a complex system. By introducing an equivalent transfer function matrix to approximate the pseudo-inverse based on the relative normalized gain, a simple decoupling internal model controller is used to solve the difficulty [16]. It is worth noting that most of the research about IMC is specific to those systems whose inverse or generalized inverse exist.

However, in engineering practice, we often encounter a class of systems in which the rank of the transfer function is often under the number of inputs or outputs. These situations are often called "rank deficiency" [17]. For example, in many kinetic reaction systems, because of linear dependence in concentration profiles coming from the mass balance or from kinetic constraints, the rank of the measurement matrix is often under the number of absorbing components [18]. It is well known that the key characteristic of the internal control strategy is to obtain the inverse controller and the internal model. However, if the characteristic of the process is rank-deficient, the design principle of internal mode control means that it is nearly impossible to apply the IMC to the rank deficiency system since the inverse of the rank deficiency system transfer function matrix does not exist. Concerning this issue, we proposed an internal model control for structured rank-deficient systems based on full rank decomposition. In this method, the system is first transformed into a column full rank system. Then a feedback-compensator is designed to improve the dynamic characteristics of the full rank system and decrease the controller design difficulty [19]. However, that method is a somewhat ideal solution, because the controlled objects cannot be decomposed in the real projects. Therefore, it is of great significance to design a direct internal mode controller for rank deficiency and to expand the IMC to the structured rank-deficient system.

In this work, an internal model control method is proposed for rank-deficient systems with time delays based on a damped pseudo-inverse. The method is obtained by first transforming the time-delay into a system without time delays by using the pole approximation approach. Then, an internal model control is designed to obtain the desired dynamic characteristics of the system. Different from our previous work [19], by introducing a damping factor here, the internal model controller is designed based on a damped pseudo-inverse, since the inverse matrix of the rank-deficient system does not exist. Furthermore, a singular value decomposition is used to analyze the steady-state performance of the system. Consequently, the selection of the damping factor is presented, and a $\mu$ analysis is made to evaluate the stability of the system. As an example, a multivariable crude distillation process was employed to verify the effectiveness of the proposed method. 


\section{Problem Statement and Preliminaries}

\subsection{System Description}

For a given structured rank-deficient system $G(s)$, the system transfer function matrix is described as:

$$
\boldsymbol{G}(s)=\left[\begin{array}{cccc}
\boldsymbol{G}_{11}(s) & \boldsymbol{G}_{12}(s) & \cdots & \boldsymbol{G}_{1 n}(s) \\
\boldsymbol{G}_{21}(s) & \boldsymbol{G}_{22}(s) & \cdots & \boldsymbol{G}_{2 n}(s) \\
\vdots & \vdots & \ddots & \vdots \\
\boldsymbol{G}_{n 1}(s) & \boldsymbol{G}_{n 2}(s) & \cdots & \boldsymbol{G}_{n n}(s)
\end{array}\right]
$$

where $G_{i j}(s)=g_{i j}(s) e^{-\tau_{i j} s},(i, j=1,2, \cdots, n)$ is a strictly rational and stable transfer function between the $j$-th input and the $i$-th output, $g_{i j}(s)$ is a transfer function, $e^{-\tau_{i j} s}$ is the time delay of $G_{i j}(s)$, and $\tau_{i j}$ is a non-negative constant. Unlike the regular process, the rank of $G(s)$ is $r$, satisfying:

$$
0<r<\min \{m, n\}
$$

The purpose of this paper is to design an internal model controller such that the closed-loop system is not only stable but also has a certain performance.

\subsection{Damped Pseudo-Inverse}

For a given matrix $A \in R^{m \times n}$, if the matrix $A$ has a full $\operatorname{rank}$ (i.e., $\operatorname{rank}(A)=\min \{m, n\}$ ), the pseudo-inverse $\boldsymbol{A}^{+} \in R^{n \times m}$ of $\boldsymbol{A}$ is:

$$
\boldsymbol{A}^{+}=\left\{\begin{array}{cc}
\left(\boldsymbol{A}^{T} \boldsymbol{A}\right)^{-1} \boldsymbol{A}^{T} & \text { if } m>n \\
\boldsymbol{A}^{-1} & \text { if } m=n \\
\boldsymbol{A}^{T}\left(\boldsymbol{A} \boldsymbol{A}^{T}\right)^{-1} & \text { if } m<n
\end{array}\right.
$$

If the matrix $A$ is rank-deficient, i.e., $\operatorname{rank}(A)<\min \{m, n\}$, a unique $A^{+}$cannot be obtained using (1), simply because of the singularity of $A^{T} A$ and $A A^{T}$ if $m \neq n$, or of the rank deficiency of $A$ if $m=n$. In the rank-deficient case, the pseudo-inverse $A_{d}{ }^{+} \in R^{n \times m}$ is expressed as:

$$
\boldsymbol{A}_{d}{ }^{+}= \begin{cases}\lim _{\rho \rightarrow 0}\left(\boldsymbol{A}^{T} \boldsymbol{A}+\rho^{2} \boldsymbol{I}\right)^{-1} \boldsymbol{A}^{T} & \text { if } m \geq n \\ \lim _{\rho \rightarrow 0} \boldsymbol{A}^{T}\left(\boldsymbol{A} \boldsymbol{A}^{T}+\rho^{2} \boldsymbol{I}\right)^{-1} & \text { if } m \leq n\end{cases}
$$

where $\rho$ is the damping factor. $\boldsymbol{A}_{d}^{+}$is called the damped pseudo-inverse of matrix $A$.

\section{Design of Decoupling Internal Model Control}

\subsection{Decoupling IMC Structure}

A block diagram of the IMC control structure is shown in Figure 1, where $\boldsymbol{R}(s), \boldsymbol{Y}(s), \boldsymbol{U}(s)$, and $\boldsymbol{P}(s)$ are the set-point values, output variables, controller output variables, and disturbance variables, respectively. $G(s)$ represents the transfer matrix of the plant with Equation (1) expression, $C(s)$ and $\boldsymbol{G}_{m}(s)$ denote the internal model controller and process model. $\boldsymbol{G}_{m}(s)$ is written as:

$$
\boldsymbol{G}_{m}(s)=\left[\begin{array}{cccc}
\boldsymbol{G}_{m 11}(s) & \boldsymbol{G}_{m 12}(s) & \cdots & \boldsymbol{G}_{m 1 n}(s) \\
\boldsymbol{G}_{m 21}(s) & \boldsymbol{G}_{m 22}(s) & \cdots & \boldsymbol{G}_{m 2 n}(s) \\
\vdots & \vdots & \ddots & \vdots \\
\boldsymbol{G}_{m n 1}(s) & \boldsymbol{G}_{m n 2}(s) & \cdots & \boldsymbol{G}_{m n n}(s)
\end{array}\right]
$$


where $\boldsymbol{G}_{m i j}(s)=g_{m i j}(s) e^{-\tau_{m i j} s},(i, j=1,2, \cdots, n)$ is a stable transfer function between the $j$-th input and the $i$-th output, $g_{m i j}(s)$ is transfer function, $e^{-\tau_{m i j} s}$ is the time delay of $\boldsymbol{G}_{m i j}(s)$, and $\tau_{m i j}$ is a non-negative constant.

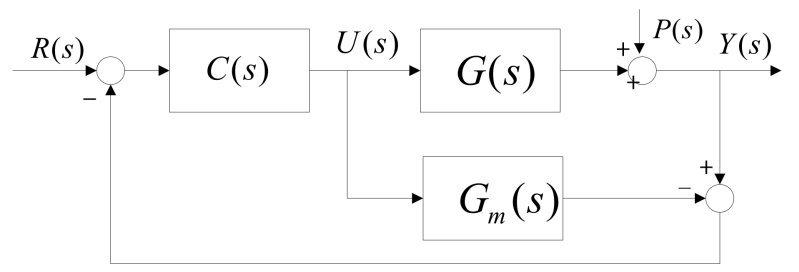

Figure 1. Basic structure of the multivariate internal model control.

Based on Figure 1, the closed-loop transfer functions from input $\boldsymbol{R}(s)$ to output $\boldsymbol{Y}(s)$ and from disturbance $\boldsymbol{P}(s)$ to $\boldsymbol{Y}(s)$ are described as follows:

$$
\begin{gathered}
\boldsymbol{G}_{R Y}(s)=\boldsymbol{Y}(s) \boldsymbol{R}^{-1}(s)=\boldsymbol{G}(s) \boldsymbol{C}(s)\left\{\boldsymbol{I}+\left[\boldsymbol{G}(s)-\boldsymbol{G}_{m}(s)\right] \boldsymbol{C}(s)\right\}^{-1} \\
\boldsymbol{G}_{P Y}(s)=\boldsymbol{Y}(s) \boldsymbol{P}^{-1}(s)=\left[\boldsymbol{I}-\boldsymbol{G}_{m}(s) \boldsymbol{C}(s)\right]\left[\boldsymbol{I}+\left[\boldsymbol{G}(s)-\boldsymbol{G}_{m}(s)\right] \boldsymbol{C}(s)\right\}^{-1}
\end{gathered}
$$

Select the ideal controller $\boldsymbol{C}(s)$ as $\boldsymbol{C}(s)=\boldsymbol{G}_{m}^{-1}(s)$, when the model of the process is perfect, that is to say when $G(s)=G_{m}(s)$, when perfect set-point tracking and disturbance rejection is achieved. In practice, however, process model mismatch is common, and the process model may not be invertible. Thus, IMC is usually designed as:

$$
\boldsymbol{C}(s)=\boldsymbol{G}_{m-}^{-1}(s) \boldsymbol{F}(s)
$$

where $\boldsymbol{G}_{m-}(s)$ is the part of minimum-phase which contains only left half plane zeros, and $\boldsymbol{F}(s)$ is a low-frequency filter to ensure the realization of $C(s)$ and attenuate the effects of a process model mismatch, formed as:

$$
\boldsymbol{F}(s)=\operatorname{diag}\left\{\frac{1}{\left(1+\lambda_{i} s\right)^{n_{i}}}\right\}, i=1,2, \cdots, n
$$

where $n_{i}$ is the filter order. We used a filter with a sufficiently high order to guarantee that the internal model controller is proper. The parameter $\lambda_{i}$ is the filter time constant, determining the response speeds.

Substituting Equation (7) into Equations (5) and (6), the previous closed-loop transfer matrixes are simplified into:

$$
\begin{gathered}
\boldsymbol{G}_{R Y}(s)=\boldsymbol{G}_{m+}(s) \boldsymbol{F}(s) \\
\boldsymbol{G}_{P Y}(s)=\boldsymbol{I}-\boldsymbol{G}_{m+}(s) \boldsymbol{F}(s)
\end{gathered}
$$

where $\boldsymbol{G}_{m+}(s)$ contains the time delay in the stable process and right half plane zeros. Letting $\boldsymbol{G}_{m+}(s)$ have the form:

$$
\boldsymbol{G}_{m+}(s)=\operatorname{diag}\left\{e^{-\theta_{K i} s} \prod_{1}^{D_{i}}\left(\frac{-\mathrm{s}+z_{p}}{s+z_{p}^{*}}\right)^{W_{i}}\right\}, \quad i=1,2, \cdots, p
$$

where $\theta_{K i}$ is the maximum predictive term in the $i$-th column of $\boldsymbol{G}_{m}^{-1}(s), z_{p}$ represents the right half-plane poles in the corresponding column, $z_{p}^{*}$ is the conjugate complex of $z_{p}, W_{i}$ represents the number of same poles in the $i$-th column of $G_{m}^{-1}(s)$, and $D_{i}$ indicates the number of distinct poles that exist in the $i$-th column of $\boldsymbol{G}_{m}^{-1}(s)$.

Then, the internal model controller is calculated by:

$$
\boldsymbol{C}(s)=\boldsymbol{G}_{m}^{-1}(s) \boldsymbol{G}_{m+}(s) \boldsymbol{F}(s)
$$




\subsection{Design of Internal Model Controller}

From the above analysis, we can see that the internal model controller is based on the inverse of the system model. As for the rank-deficient system $G(s)$, the inverse of the matrix $G^{-1}(s)$ doesn't exist. That is to say, a traditional IMC cannot solve the control problem of the rank-deficient system directly. He [20] uses a pseudo inverse constant to set the input delay of different channels, and designs a delay controller to adjust the input and output so as to achieve a satisfactory control effect. In this subsection, our purpose is to obtain the internal model controller $C(s)$ based on the damped pseudo-inverse $G_{\mathrm{d}}{ }^{+}(s)$ with an improved system time delay by using the time delay approximation method.

Suppose the model of the process is perfect, the damped pseudo-inverse of the process model $\boldsymbol{G}_{d}^{+}(s)$ is:

$$
\boldsymbol{G}_{d}{ }^{+}(s)=\lim _{\rho \rightarrow 0} \boldsymbol{G}_{m}^{T}(s)\left(\boldsymbol{G}_{m}(s) \boldsymbol{G}_{m}^{T}(s)+\rho^{2} \boldsymbol{I}\right)^{-1}
$$

Then, the internal model controller $\boldsymbol{C}(s)$ is designed as:

$$
C_{d}(s)=G_{d}^{+}(s) G_{d+}(s) F(s)
$$

where $G_{d+}(s)$ is the non-minimum-phase, with the form of:

$$
\boldsymbol{G}_{d+}(s)=\operatorname{diag}\left\{e^{-\theta_{K i} s} \prod_{1}^{D_{i}}\left(\frac{-\mathrm{s}+z_{p}}{s+z_{p}^{*}}\right)^{W_{i}}\right\}, \quad i=1,2, \cdots, p
$$

where $\theta_{K i}$ is the maximum predictive term in the $i$-th column of $\boldsymbol{G}_{d}^{+}(s), z_{p}$ represents the right half-plane poles in the corresponding column, $z_{p}^{*}$ is the conjugate complex of $z_{p}, W_{i}$ represents the number of same poles in the $i$-th column of $G_{d}^{+}(s)$, and $D_{i}$ indicates the number of distinct poles that exist in the $i$-th column of $G_{d}^{+}(s)$.

Since the rank-deficient system $G(s)$ contains time delays, $G_{i j}(s)=g_{i j}(s) e^{-\tau_{i j} s}$, it is difficult to realize and calculate the controller $C_{d}(s)$. To overcome the difficulties, an approximation is used to approximate the time delay term $e^{-\tau_{i j} s}$ [21]. It was indicated that the Pade approximation is a very effective way to deal with time delay. In this work, we adopt a first-order Pade approximation to approximate $e^{-\tau_{i j} j^{s}}$, which is expressed as:

$$
e^{-\tau_{i j} s}=\frac{1-0.5 \tau_{i j} s}{1+0.5 \tau_{i j} s}
$$

After the approximation, the order of the controller $C_{d}(s)$ will become very high, which is difficult to realize in engineering practices. Due to this, we can use a low-order model with a rational transfer function to approximate the controller. Here, we used the Pade approximation, because in chemical processes most of the objects can be described by the first- or second-order plus the pure lag link. The low-order model is expressed as $C_{d}{ }^{r}(s)$, after which the closed-loop transfer matrix will turn out to be:

$$
\boldsymbol{G}_{R Y}(s)=\boldsymbol{G}(s) \boldsymbol{C}_{d}^{r}(s) \boldsymbol{F}(s)
$$

One convenient way to parameterize low-rank matrices is via the singular value decomposition (SVD). We will use SVD to analyze the steady-state performance in the next section.

\subsection{Steady-State Performance Analysis by SVD}

In modern control theory, singular values have been used to extend the classical frequency response Bode plot to multivariable systems. To consider the system (1), based on Figure 1, the system output can be described by:

$$
\boldsymbol{Y}(s)=\boldsymbol{G}(s) \boldsymbol{U}(s)
$$


Frequency responses at $s=j w$ are given as:

$$
\boldsymbol{Y}(j w)=\boldsymbol{G}(j w) \boldsymbol{U}(j w)
$$

After the singular value decomposition, the controlled system matrix $G(j w)$ can be written as:

$$
\boldsymbol{G}(j w)=\boldsymbol{W}(j w) \boldsymbol{D}(j w) \boldsymbol{V}^{T}(j w)
$$

where $\boldsymbol{W}(j w) \in R^{m \times m}$ is the matrix of left singular vectors. $\boldsymbol{D}(j w)$ is a diagonal matrix whose elements are zero except for the first $r$ (rank of $G(j w)$ ) elements along the main diagonal. These are the non-zero singular values of $\boldsymbol{G}_{m}(0) . \boldsymbol{V}(j w) \in R^{n \times n}$ is the matrix of right singular vectors. $\boldsymbol{W}(j w)$ and $\boldsymbol{V}(j w)$ are unitary matrices.

For calculation conveniences, we choose the state when the system is stable, namely $j w=0$. Letting $\boldsymbol{W}(0)=\left[w_{1}, w_{2}, \cdots, w_{m}\right], \boldsymbol{V}(0)=\left[v_{1}, v_{2}, \cdots, v_{n}\right], \boldsymbol{D}(0)$ is formed as:

$$
\boldsymbol{D}(0)=\left[\begin{array}{ll}
S & 0 \\
0 & 0
\end{array}\right] \in R^{m \times n}
$$

where $S=\operatorname{diag}\left\{e_{1}, e_{2}, \cdots, e_{r}\right\}$, and $e_{1} \geq e_{2} \geq \cdots \geq e_{r}$ are non-zero singular values. Then, $G(0)$ is expressed as:

$$
G(0)=e_{1} w_{1} v_{1}^{T}+e_{2} w_{2} v_{2}^{T}+\cdots+e_{r} w_{r} v_{r}^{T}=\sum_{i=1}^{r} e_{i} w_{i} v_{i}^{T}
$$

Then, the pseudo-inverse of $\boldsymbol{G}_{m}(0)$ is:

$$
\boldsymbol{G}_{m}{ }^{+}(0)=\boldsymbol{V D}^{+} \boldsymbol{W}^{T}=\sum_{i=1}^{r} \frac{1}{e_{i}} v_{i} w_{i}^{T}
$$

The corresponding damped pseudo-inverse $\boldsymbol{G}_{m d}{ }^{+}(0)$ is obtained as:

$$
\boldsymbol{G}_{m d}{ }^{+}(0)=\boldsymbol{G}_{m}{ }^{T}(0)\left(\boldsymbol{G}_{m}(0) \boldsymbol{G}_{m}{ }^{T}(0)+\rho^{2} \boldsymbol{I}\right)^{-1}=\sum_{i=1}^{r} \frac{e_{i}}{e_{i}^{2}+\rho^{2}} v_{i} \mathbf{w}_{i}^{T}
$$

It is well known that $\boldsymbol{U}(0)$ is a least-squares solution to (18), i.e.,

$$
\boldsymbol{U}(0)=\boldsymbol{Y}(0) \boldsymbol{G}^{+}(0)
$$

satisfies

$$
\arg \min _{U(0)}\|\boldsymbol{Y}(0)-\boldsymbol{G}(0) \boldsymbol{U}(0)\|_{2}
$$

Equation (23) indicates that:

$$
\left\|\boldsymbol{C}_{d}(s)\right\|_{2}=\left\|\boldsymbol{G}_{m d}^{+}(s) \boldsymbol{G}_{m+}(s) \boldsymbol{F}(s)\right\|_{2}<\left\|\boldsymbol{G}_{m}^{+}(s) \boldsymbol{G}_{m+}(s) \boldsymbol{F}(s)\right\|_{2}=\|\boldsymbol{C}(s)\|_{2}
$$

The system steady-state error $\boldsymbol{E}(0)$ is:

$$
\boldsymbol{E}(0)=\boldsymbol{R}(0)-\boldsymbol{Y}(0)=\left(\boldsymbol{I}-\boldsymbol{G}(0) \boldsymbol{C}_{d}^{r}(0)\right) \boldsymbol{R}(0)
$$

It is obvious that:

$$
\|\boldsymbol{E}(0)\|_{2}=\left\|\boldsymbol{I}-\boldsymbol{G}(0) \boldsymbol{G}_{d}{ }^{+}(0) \boldsymbol{G}_{d+}(0) \boldsymbol{F}(0)\right\|_{2} \geq\left\|\boldsymbol{I}-\boldsymbol{G}(0) \boldsymbol{G}_{m}{ }^{+}(0) \boldsymbol{G}_{m+}(0) \boldsymbol{F}(0)\right\|_{2}
$$


Since the system $G(s)$ is rank-deficient, the least-squares solutions of Equation (18) are non-unique. In order to obtain a unique solution, an additional minimum norm condition $\boldsymbol{U}^{T}(0) \boldsymbol{U}(0)=\operatorname{argmin}$ is needed with respect to certain decision variables, namely:

$$
\operatorname{argmin}\|\boldsymbol{U}(0)\|_{2}
$$

From Equations (26) and (28), we can see that, though $C_{d}(s)$ decreased the value of $\|U(s)\|$, it increased $\|\boldsymbol{E}(s)\|$. Hence, we can set $\rho^{2}$ as a decision variable, and design the controller by selecting the appropriate $\rho^{2}$ to realize compromises between Equations (24) and (29). Usually, $\rho^{2}$ satisfies the following relationship:

$$
\rho^{2}=\left\{\begin{array}{cc}
\rho_{0}^{2}\left(1-e_{r} / \varepsilon_{r}\right)^{2} & 0 \leq e_{r} \leq \varepsilon_{r} \\
0 & e_{r}>\varepsilon_{r}
\end{array}\right.
$$

where $\rho_{0}$ is the maximum damping factor and can be selected through simulations, and $\varepsilon_{r}$ is a singular measure, which is defined as:

$$
\varepsilon_{r}=\frac{e_{1}}{e_{r}}
$$

\section{Robust Analysis}

Modeling errors and system uncertainties are inevitable in the actual projects. The presence of uncertainties usually causes instability and the poor performance of control systems. It is therefore necessary to analyze the robust stability of the proposed control system. In order to evaluate the robustness of the controllers, a structured singular-value analysis is carried out in the presence of diagonal additive uncertainty. Suppose that the control system with the additive uncertainty is denoted by:

$$
G(s)=G_{m}(s)+\Delta(s)
$$

where $\Delta(s)=\operatorname{diag}\left\{\Delta_{i}(s), i=1,2, \cdots, m\right\}$ is the structured uncertainty weight, which satisfies:

$$
\Delta_{i}(s)=\boldsymbol{T}_{i}(s) \boldsymbol{\theta}_{i}(s) \boldsymbol{L}_{i}(s)
$$

where $\boldsymbol{T}_{i}(s)$ and $\boldsymbol{L}_{i}(s)$ are constant transfer matrices, and $\boldsymbol{\theta}_{i}(s)$ is any stable transfer matrix from a set satisfying $\|\boldsymbol{\theta}(j \omega)\| \leq 1, \forall \omega$. Figure 2 describes the control system with the additive uncertainty.

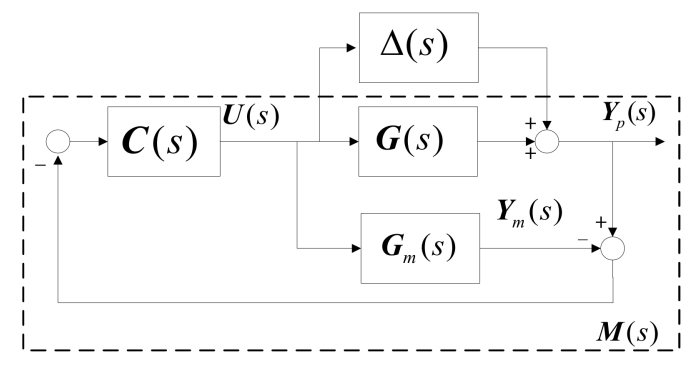

Figure 2. System with additive uncertainty and its $M-\Delta$ structure.

To conveniently analyze the robust stability, the general $M-\Delta$ structure is achieved in Figure 2. For the nominal system, the matrix $-\boldsymbol{M}(s)$ is equivalent to the closed loop transfer function, i.e., $\boldsymbol{M}(s)=-\boldsymbol{G}_{R Y}(s)$. The structured singular-value of $\boldsymbol{M}(s)$ is defined as:

$$
\mu(\boldsymbol{M})^{-1}=\min _{\Delta}\{\bar{\sigma}(\Delta) \mid \operatorname{det}(I-\boldsymbol{M} \Delta)=0\}
$$

where $\bar{\sigma}(\Delta)$ denotes the maximum singular value. 
Theorem 1. Consider the feedback control system shown in Figure 2, the feedback loop with the plant $G(s)$ and controller $\boldsymbol{C}(s)$ will remain stable in the face of all possible perturbed uncertainties $\Delta(s)$, if and only if:

$$
\mu(\boldsymbol{M}(j w))<1
$$

Proof. If the system is unstable in the face of all possible perturbed uncertainties $\Delta(s), \operatorname{det}(I-M \Delta(j w))$ will surround the origin of the complex plane. At the critical stability, $\operatorname{det}(I-M \Delta(j w))=0$, namely $\underline{\sigma}(I-\boldsymbol{M} \Delta(j w))=0$. That is to say, the system is still stable after being perturbed if $\underline{\sigma}(I-\boldsymbol{M} \Delta(j w))>0$. From the property of $\sigma(I-\boldsymbol{M} \Delta(j w))>I-\bar{\sigma}(\boldsymbol{M} \Delta(j w))$, it can be deduced that the system is stable after being perturbed if $\bar{\sigma}(\boldsymbol{M} \Delta(j w))<1$. Using singular value inequality:

$$
\bar{\sigma}(\boldsymbol{M} \Delta(j w)) \leq \bar{\sigma}(\boldsymbol{M}(j w)) \bar{\sigma}(\Delta(j w))
$$

we can see that $\bar{\sigma}(\boldsymbol{M} \Delta(j w))<1$, provided $\bar{\sigma}(\boldsymbol{M}(j w))<[\bar{\sigma}(\Delta(j w))]^{-1}$. According to the robust stability theorem, the proposed control system is robustly stable for $\Delta(s)$ if and only if $\bar{\sigma}(M(\mathrm{jw}))<1, \quad \forall \omega$. Hence, when $[\bar{\sigma}(\Delta(j w))]^{-1}<1$, the proposed control system is robustly stable. Namely, $\mu(\boldsymbol{M}(j w))<1$. This completed the proof.

If $\Delta(s)$ is known, Equation (36) can be employed to evaluate the robust stability of the proposed control system. In other words, if the magnitude plots of the left side of (36) are under 1 at $\forall \omega$, the system can be considered robustly stable.

\section{Simulation}

To demonstrate the effectiveness of the proposed method, we considered a crude distillation process with 5 inputs and 4 outputs. The process transfer function matrix is given below [22]:

$$
G(\mathrm{~s})=\left[\begin{array}{ccccc}
\frac{3.8(16 \mathrm{~s}+1)}{140 \mathrm{~s}^{2}+14 \mathrm{~s}+1} & \frac{2.9 \mathrm{e}^{-6 \mathrm{~s}}}{10 \mathrm{~s}+1} & 0 & 0 & \frac{0.73(-16 \mathrm{~s}+1) \mathrm{e}^{-4 \mathrm{~s}}}{150 \mathrm{~s}^{2}+20 \mathrm{~s}+1} \\
\frac{3.9(4.5 \mathrm{~s}+1)}{96 \mathrm{~s}^{2}+14 \mathrm{~s}+1} & \frac{6.3}{20 \mathrm{~s}+1} & 0 & 0 & \frac{16 \mathrm{~s}^{-2 \mathrm{~s}}}{(5 \mathrm{~s}+1)(14 \mathrm{~s}+1)} \\
\frac{3.8(0.8 \mathrm{~s}+1)}{23 \mathrm{~s}^{2}+13 \mathrm{~s}+1} & \frac{6.1(12 \mathrm{~s}+1) \mathrm{e}^{-\mathrm{s}}}{337 \mathrm{~s}^{2}+34 \mathrm{~s}+1} & \frac{3.4 \mathrm{e}^{-2 \mathrm{~s}}}{6.9 \mathrm{~s}+1} & 0 & \frac{22 \mathrm{se}}{(5 \mathrm{~s}+1)(10 \mathrm{~s}+1)} \\
\frac{-1.62(5.3 \mathrm{~s}+1) \mathrm{e}^{-\mathrm{s}}}{13 \mathrm{~s}^{2}+13 \mathrm{~s}+1} & \frac{-1.53(3.1 \mathrm{~s}+1)}{5.1 \mathrm{~s}^{2}+7.1 \mathrm{~s}+1} & \frac{-1.37 .6 \mathrm{~s}+1)}{4.7 \mathrm{~s}^{2}+7.1 \mathrm{~s}+1} & \frac{-0.6 \mathrm{e}^{-\mathrm{s}}}{2 \mathrm{~s}+1} & \frac{0.32(-9.1 \mathrm{~s}+1) \mathrm{e}^{-\mathrm{s}}}{12 \mathrm{~s}^{2}+15 \mathrm{~s}+1}
\end{array}\right]
$$

Designing the internal model controller using the method proposed in a previous work [23], the output responses of the system for the given step inputs $r_{1}=1, r_{2}=1.2, r_{3}=1.5$, and $r_{4}=2$ are shown in Figure 3.

Assume that the fourth channel becomes a linear relationship of the other three channels because a failure happens on it. That is to say,

$$
y_{4}=k_{1} y_{1}+k_{2} y_{2}+k_{3} y_{3}
$$

Let $k_{1}=0.3, k_{2}=0.3$, and $k_{3}=0.25$, and let $\rho=0.01$, from Equation (23) we can get the internal model controller as:

$$
C_{d}^{r}(\mathrm{~s})=\left[\begin{array}{cccc}
\frac{0.444}{30 \mathrm{~s}+1} & -\frac{0.2024}{\mathrm{~s}+1} & \frac{0.0255}{\mathrm{~s}+1} & \frac{0.066}{\mathrm{~s}+1} \\
-\frac{0.2751}{30 \mathrm{~s}+1} & \frac{0.284}{\mathrm{~s}+1} & -\frac{0.0158}{\mathrm{~s}+1} & -\frac{0.0408}{\mathrm{~s}+1} \\
-\frac{0.0031}{30 \mathrm{~s}+1} & -\frac{0.2834}{\mathrm{~s}+1} & \frac{0.2939}{\mathrm{~s}+1} & -\frac{0.005}{\mathrm{~s}+1} \\
-\frac{0.5713}{30 \mathrm{~s}+1} & \frac{0.476}{\mathrm{~s}+1} & -\frac{0.6281}{\mathrm{~s}+1} & -\frac{1.643}{\mathrm{~s}+1} \\
-\frac{0.1493}{30 \mathrm{~s}+1} & \frac{0.0749}{\mathrm{~s}+1} & \frac{0.0699}{\mathrm{~s}+1} & \frac{0.1812}{\mathrm{~s}+1}
\end{array}\right]
$$




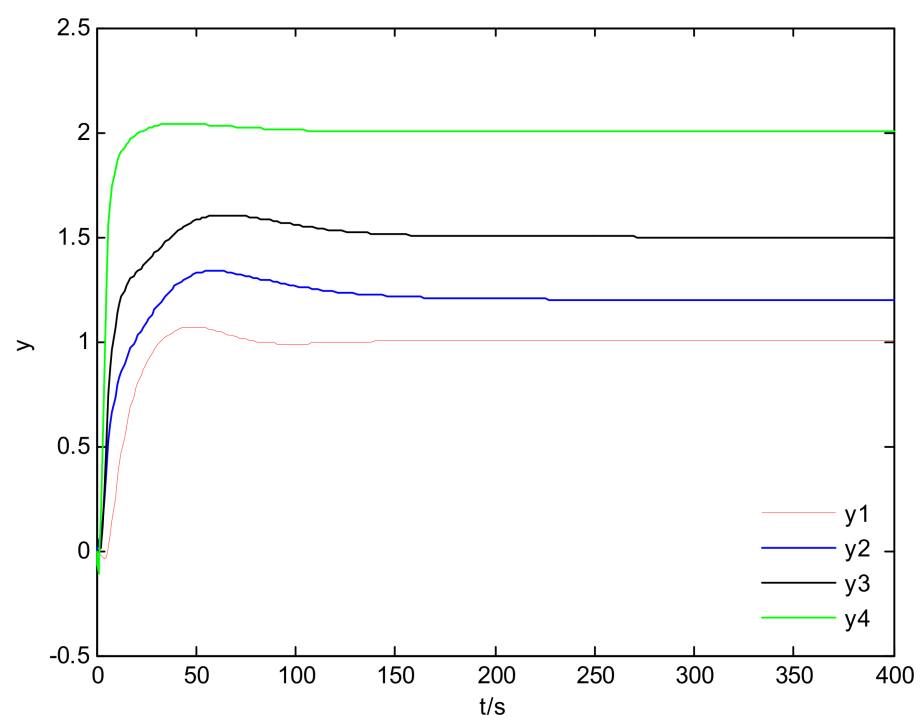

Figure 3. Output responses of the normal system.

The simulation results are shown in Figures 4-7. It can be seen from the simulation results that the method proposed in this work can track the system setting value with a smaller tracking error when the unit-step response of the non-fault channel is carried out. However, for the fault channel, the method proposed in this paper showed an obvious weakness, though the tracking error is smaller than the original method.
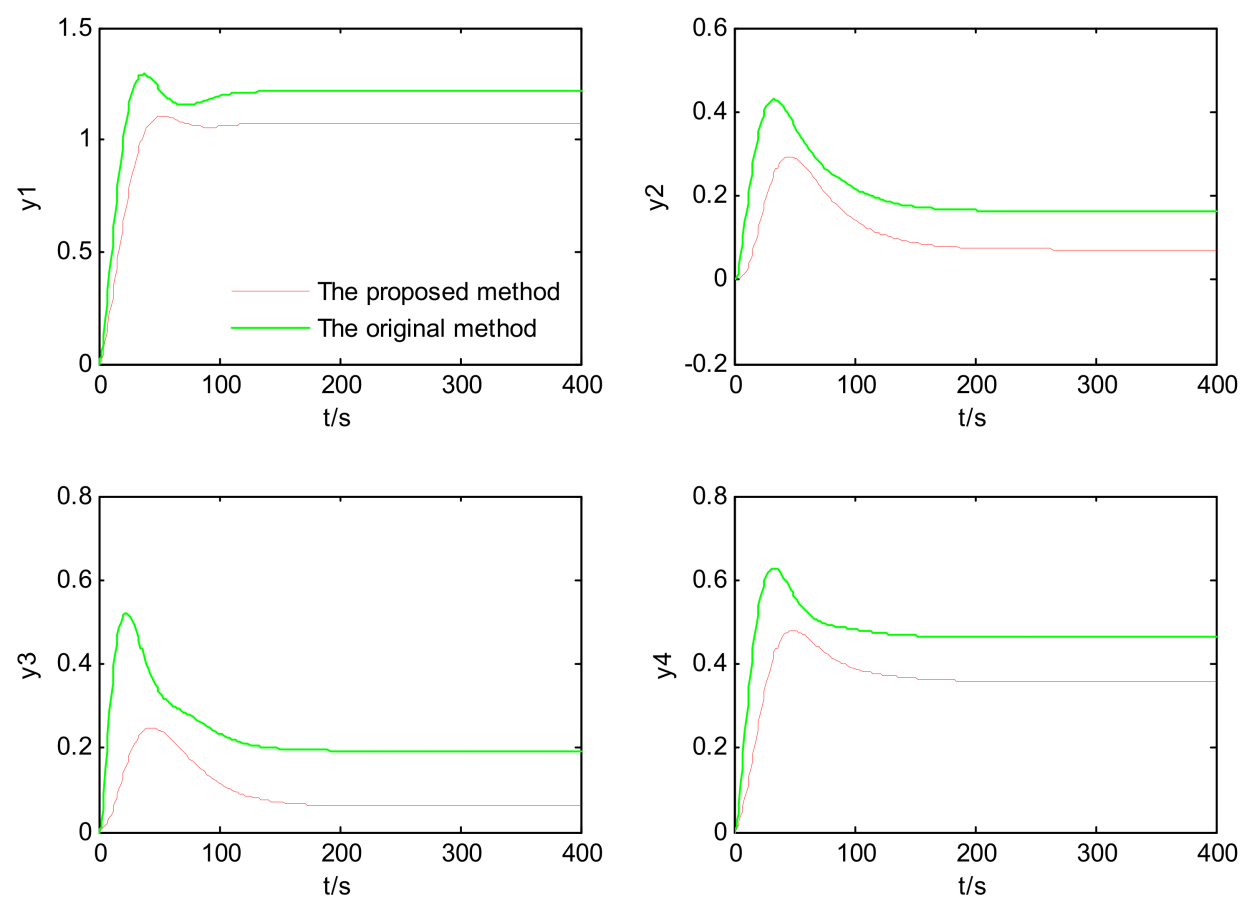

Figure 4. Set-point responses of $r_{1}=1$ and $r_{2}=r_{3}=r_{4}=0$. 

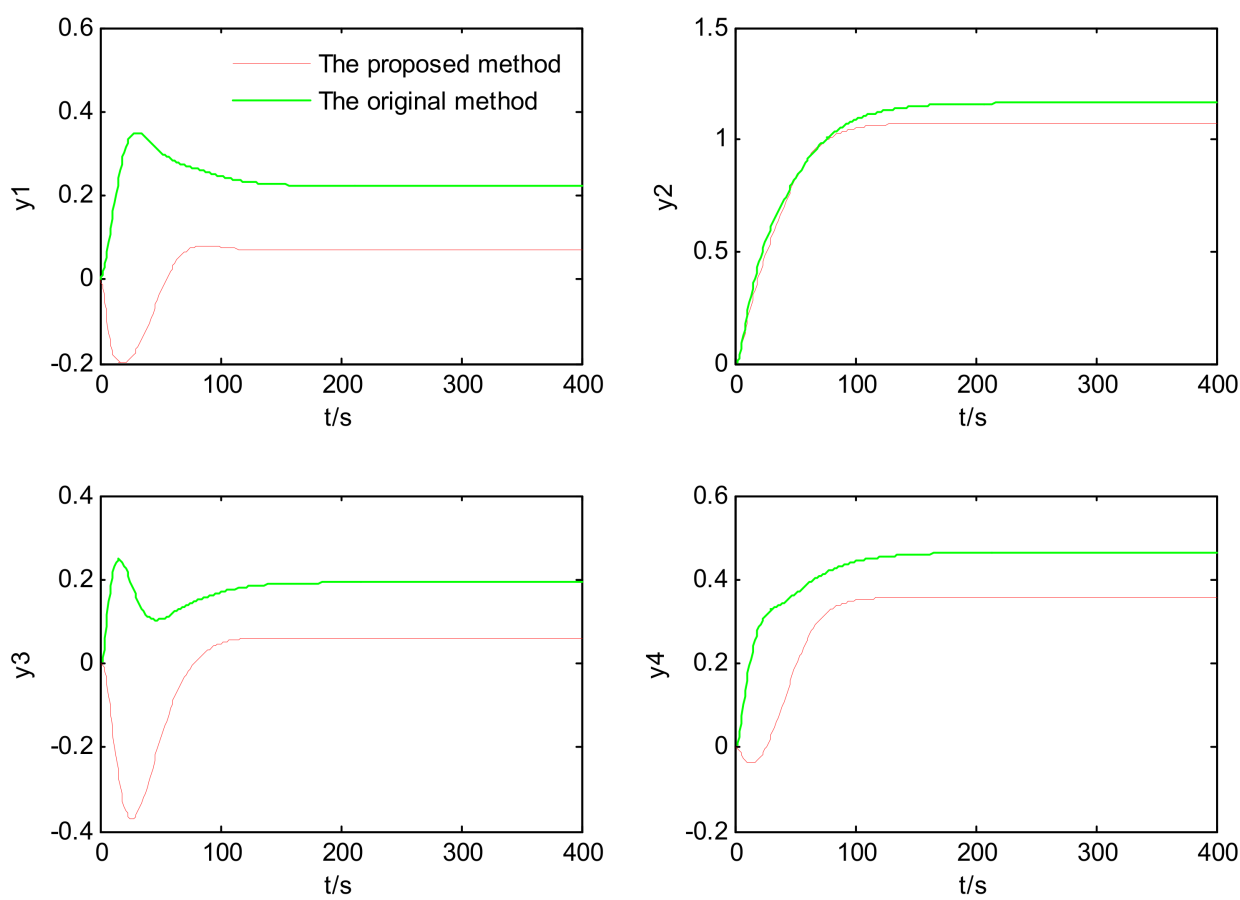

Figure 5. Set-point responses of $r_{2}=1$ and $r_{1}=r_{3}=r_{4}=0$.
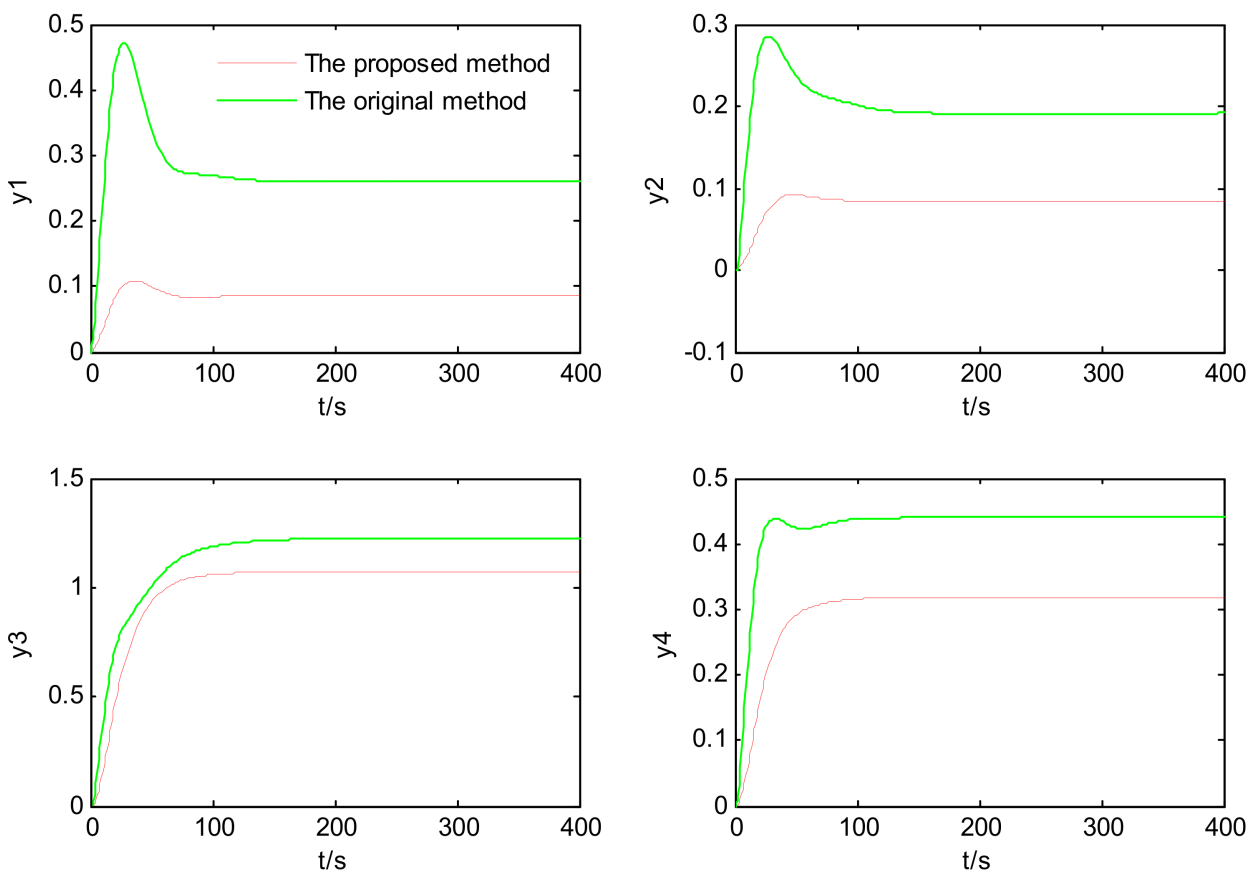

Figure 6. Set-point responses of $r_{3}=1$ and $r_{1}=r_{2}=r_{4}=0$. 

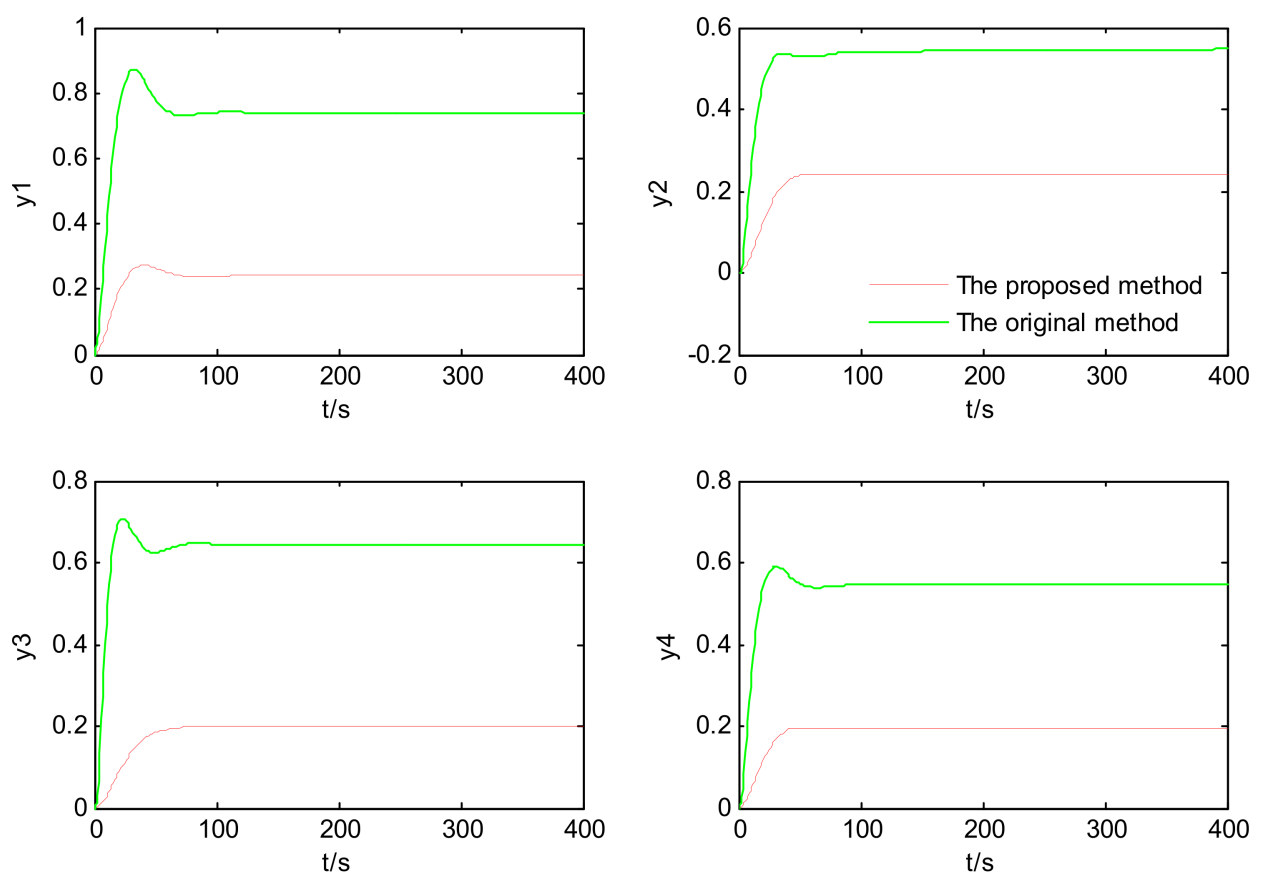

Figure 7. Set-point responses of $r_{4}=1$ and $r_{2}=r_{3}=r_{4}=0$.

The ISE (Integral Square Error) index is used to demonstrate the superiority of the proposed method. For multivariable systems, the calculation formula of the ISE index is:

$$
I S E=\sum_{i=1}^{4} I S E_{y_{i}}
$$

where $I S E_{y i}=I S E_{y i-r 1}+I S E_{y i-r 2}+I S E_{y i-r 3}+I S E_{y i-r 4}$.

Due to the steady-state deviation of the fault system, the calculation formula needs to be modified when calculating the ISE value. In order to fully consider the steady state performance of the system, this work integrated the time before the deviation stabilized. From the above analysis shown on the figures, we can take $T$ as 350 s, namely:

$$
\mathrm{ISE}_{y i-r i}=\int_{0}^{T}\left(1-y_{i}(t)\right)^{2} d t, \mathrm{ISE}_{y i-r j}=\int_{0}^{T}\left(0-y_{i}(t)\right)^{2} d t \quad(i \neq j)
$$

The ISE values of the system are shown in Table 1.

As can be seen from Table 1 , when $r_{1}=1, r_{2}=1, r_{3}=3$, and $r_{4}=1$ are adopted, the ISE index error of the proposed method is much smaller than that of the original method, which proves that the effect of the proposed method in this work has a better tracking performance than the original method.

We assume that the fault channel of the system is a linear combination of the normal channels, so the value of $k_{i}(i=1,2,3)$ will directly affect the performance of the system. In order to better analyze this feature, we also take the ISE value of the system as the index. We fixed $k_{1}, k_{2}$, and $k_{3}$ respectively and studied the impact of the other two parameters on the system. 
Table 1. Comparison of the ISE values.

\begin{tabular}{cccccc}
\hline$r_{1}=1$ & ISE11 & ISE21 & ISE31 & ISE41 & ISE1 \\
\hline Proposed method & 13.9462 & 6.0384 & 4.3918 & 47.7656 & 72.1419 \\
Original method & 23.2320 & 16.8642 & 21.8216 & 79.7855 & 141.7032 \\
\hline$r_{2}=1$ & ISE12 & ISE22 & ISE32 & ISE42 & ISE2 \\
\hline Proposed method & 2.5843 & 20.2293 & 4.8419 & 36.9471 & 64.6026 \\
Original method & 20.2013 & 23.2482 & 11.4306 & 65.6133 & 120.4934 \\
\hline$r_{3}=3$ & ISE13 & ISE23 & ISE33 & ISE43 & ISE3 \\
\hline Proposed method & 2.5183 & 2.3878 & 17.7328 & 31.9622 & 54.6012 \\
Original method & 27.8294 & 14.1794 & 23.2003 & 64.3888 & 129.5978 \\
\hline$r_{4}=1$ & ISE14 & ISE24 & ISE34 & ISE44 & ISE4 \\
\hline Proposed method & 19.7096 & 18.7629 & 12.7664 & 233.1242 & 284.3630 \\
Original method & 189.1030 & 97.9024 & 141.3680 & 78.6363 & 507.0097 \\
\hline
\end{tabular}

From Figures 8-10, we can see that when $k_{1}, k_{2}$, and $k_{3}$ change, the ISE values of the system also vary accordingly. Through a comprehensive analysis of these three figures, it is not difficult to find that when one value gradually increases, the other two values gradually decrease, which will reduce the system error. When the values of the three parameters meet the requirements, $\sum_{i=1}^{3} k_{i}=1$, the ISE value is the minimum and the control effect is the best.
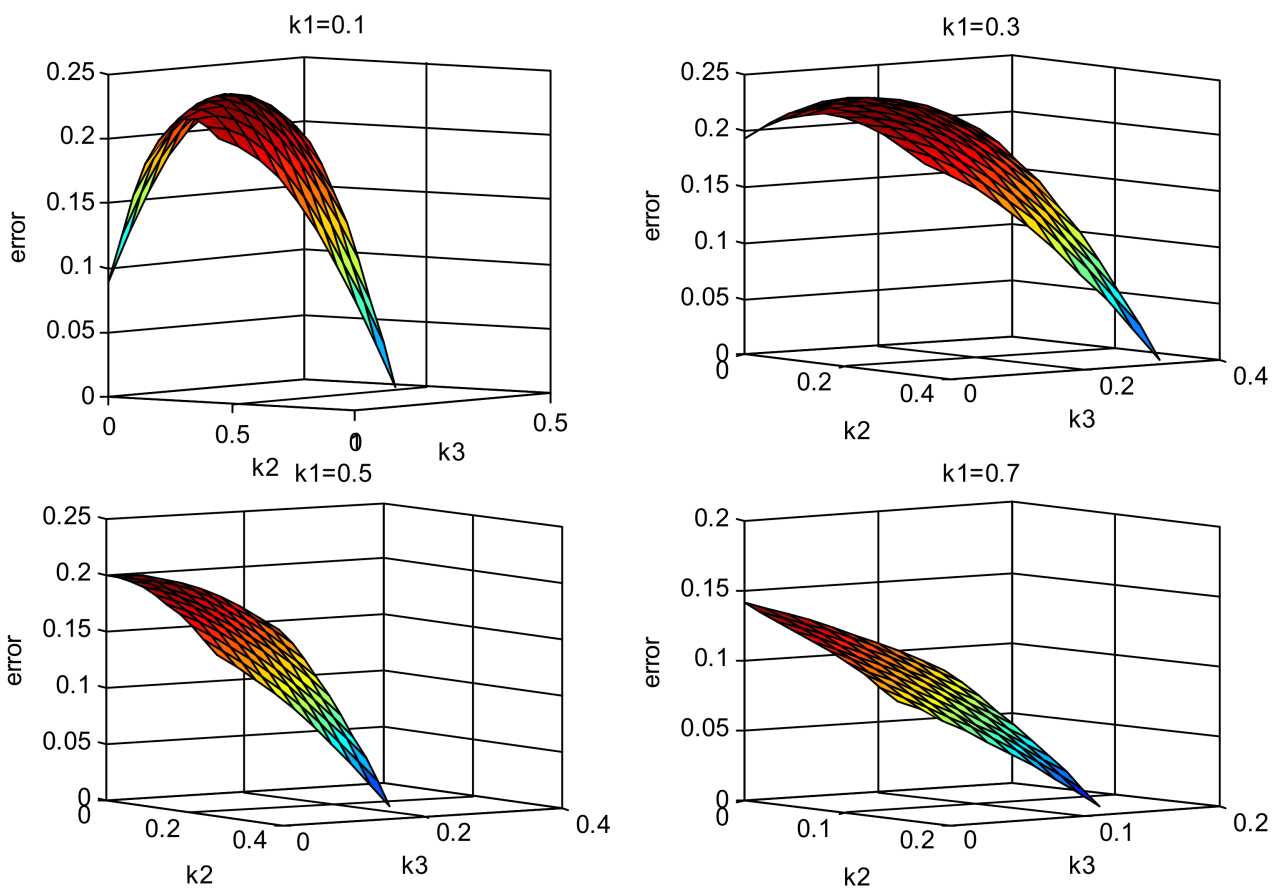

Figure 8. Effect of $k_{2}$ and $k_{3}$ on the system ISE value for different $k_{1}$ values. 

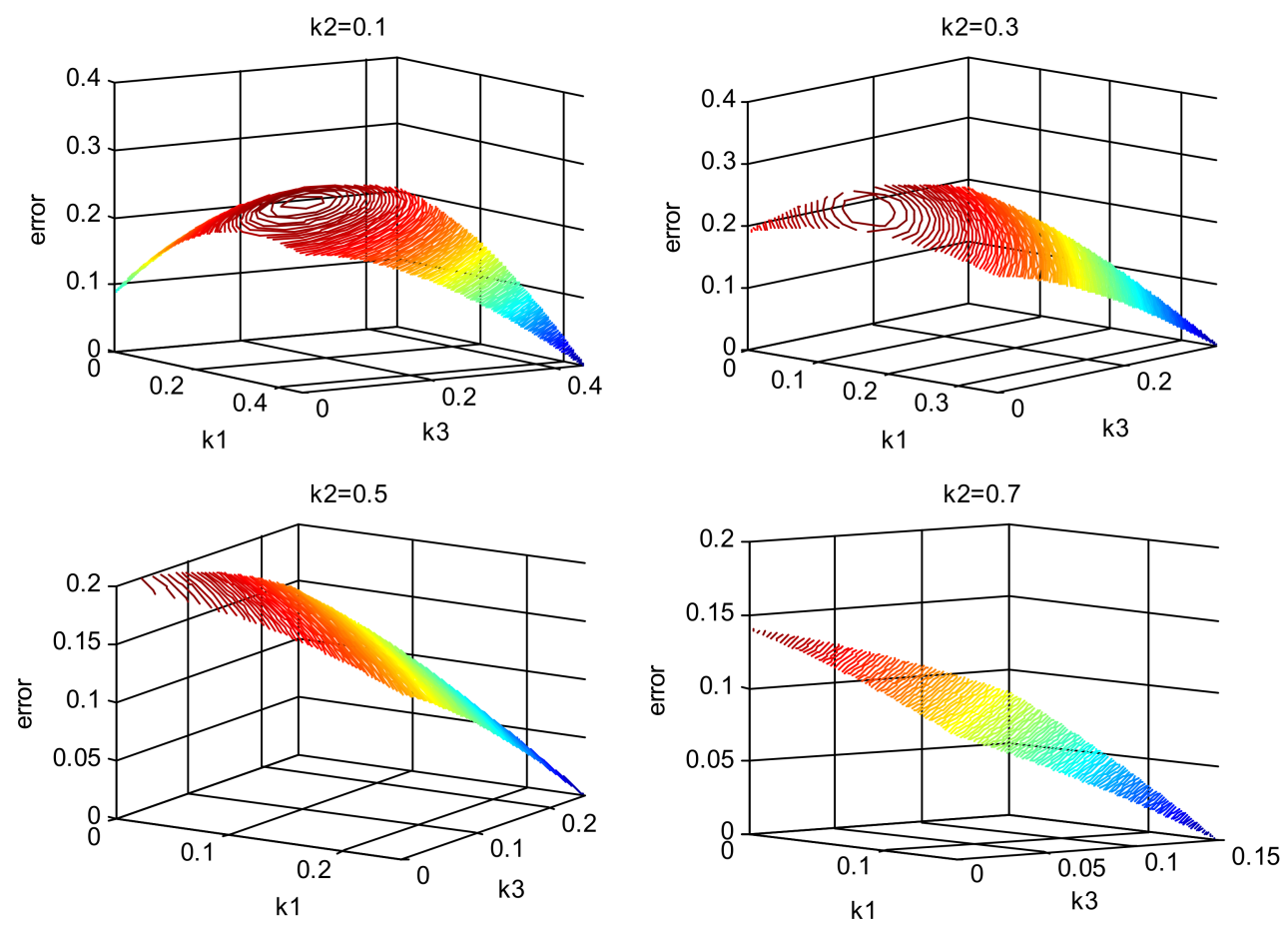

Figure 9. Effects of $k_{1}$ and $k_{3}$ on the system ISE value for different $k_{2}$ values.
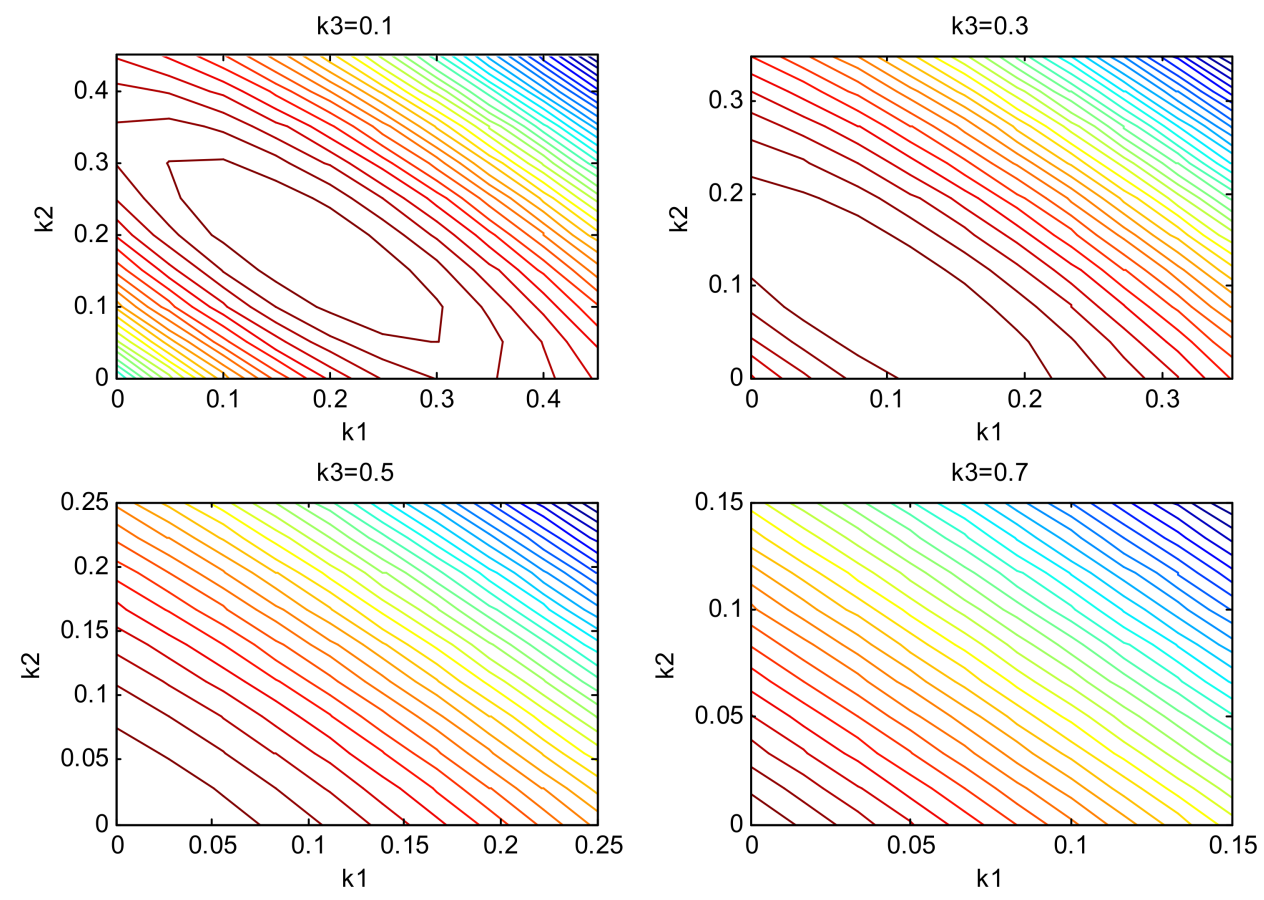

Figure 10. Effect of $k_{1}$ and $k_{2}$ on the system ISE value for different $k_{3}$ values.

\section{Conclusions}

In this work, an internal model control method is first proposed for rank-deficient systems with time delays based on a damped pseudo-inverse. The method is obtained by first transforming the time-delay into a system without time delays, using the pole approximation approach. Then, an internal model control is designed to obtain the desired dynamic characteristics of the system. It is well known that the design of the internal model controller is based on the inverse of the system transfer function matrix. By introducing a damping factor, the internal model controller is designed based 
on a damped pseudo-inverse, since the inverse matrix of the rank-deficient system does not exist. Furthermore, a singular value decomposition is used to analyze the steady-state performance of the system. Consequently, the selection of the damping factor is presented. In the end, the method of the $\mu$ analysis is used to study the stability of the system. The simulations results illustrate that not only does the proposed strategy guarantee the system's stability, but it also has a certain dynamic performance.

However, there are several limitations in our present study, which should be taken into consideration. First, we transform the time-delays system into a system without time delays, which might increase the system error. Another limitation is that the experiments were conducted in a system with an assumed fault channel: whether it can still guarantee a desired performance in terms of a real rank deficiency still needs to be confirmed. Finally, an additional problem that should be tackled is how to extend this method to perturbed systems. In spite of these limitations, we believe that this study represents a useful step toward developing a control method for rank-deficient systems.

Author Contributions: M.J. carried out the simulations and wrote the paper. B.J. developed the model and designed the article. Q.W. contributed to the discussion and provided improving suggestions. All authors have read and approved the final manuscript.

Funding: This research was funded by the Premium Funding Project for Academic Human Resources Development in Beijing Union University (No. BPHR2018DZ05), and the Social Science Foundation of Beijing (No. 15JGC188).

Acknowledgments: The authors are grateful to the anonymous reviewers for their valuable recommendations.

Conflicts of Interest: The authors declare no conflict of interest.

\section{References}

1. Albertos, P.; Sala, A.; Chadli, M. Multivariable control systems-An engineering approach. Automatica 2005, 41, 1665-1666. [CrossRef]

2. Hunek, W.P.; Latawiec, K.J. Minimum variance control of discrete-time and continuous-time lti mimo systems-A new unified framework. Control Cybern. 2009, 38, 609-624.

3. Ortiz, J.P.; Minchala, L.I.; Reinoso, M.J. Nonlinear robust h-infinity pid controller for the multivariable system quadrotor. IEEE Lat. Am. Trans. 2016, 14, 1176-1183.

4. Karimi, A.; Kammer, C. A data-driven approach to robust control of multivariable systems by convex optimization. Automatica 2017, 85, 227-233. [CrossRef]

5. Xavier, N.; Bandyopadhyay, B.; Schmid, R. Robust non-overshooting tracking using continuous control for linear multivariable systems. IET Control Theory Appl. 2018, 12, 1006-1011. [CrossRef]

6. Savran, A. A multivariable predictive fuzzy pid control system. Appl. Soft Comput. 2013, 13, $2658-2667$. [CrossRef]

7. Castillo, O.; Cervantes, L.; Melin, P.; Pedrycz, W. A new approach to control of multivariable systems through a hierarchical aggregation of fuzzy controllers. Granul. Comput. 2019, 4, 1-13. [CrossRef]

8. Garcia, C.E.; Morari, M. Internal model control. A unifying review and some new results. Ind. Eng. Chem. Process Des. Dev. 1982, 21, 308-323. [CrossRef]

9. Yao, Y.; Wang, J.; Pan, L. Decoupling internal model control for multi-variable non-square system with time delays. J. Chem. Ind. Eng. 2008, 59, 1737-1742.

10. Chekari, T.; Mansouri, R.; Bettayeb, M. Imc-pid fractional order filter multi-loop controller design for multivariable systems based on two degrees of freedom control scheme. Int. J. Control Autom. Syst. 2018, 16, 689-701. [CrossRef]

11. Garcia, C.E.; Morari, M. Internal model control. 2. Design procedure for multivariable systems. Ind. Eng. Chem. Process Des. Dev. 1985, 24, 472-484. [CrossRef]

12. Tan, G.T.; Chiu, M.S. A multiple-model approach to decentralized internal model control design. Chem. Eng. Sci. 2001, 56, 6651-6660. [CrossRef]

13. Tang, Y.; Du, F. A delay compensation approach based on internal model control for two-input two-output networked control systems. Clust. Comput. 2018, 1-12. [CrossRef]

14. Garrido, J.; Vazquez, F.; Morilla, F. Inverted decoupling internal model control for square stable multivariable time delay systems. J. Process Control 2014, 24, 1710-1719. [CrossRef] 
15. Chen, P.Y.; Ou, L.L.; Sun, J.; Zhang, W.D. Modified internal model control and its application in non-square processes. Control Decis. 2008, 23, 581-584.

16. Rajapandiyan, C.; Chidambaram, M. Controller design for mimo processes based on simple decoupled equivalent transfer functions and simplified decoupler. Ind. Eng. Chem. Res. 2012, 51, 12398-12410. [CrossRef]

17. de Juan, A.; Navea, S.; Diewok, J.; Tauler, R. Local rank exploratory analysis of evolving rank-deficient systems. Chemom. Intell. Lab. Syst. 2004, 70, 11-21. [CrossRef]

18. Xu, C.J.; Gourvenec, S.; Liang, Y.Z.; Massart, D.L. Local factor analysis of rank-deficient reaction systems. Anal. Chim. Acta 2006, 575, 1-8. [CrossRef]

19. Jiang, M.Y.; Jiang, B.Y.; Cai, W.; Du, X.H.; Zhang, Y.M.; Huang, Y. Internal model control for structured rank deficient system based on full rank decomposition. Clust. Comput. 2017, 20, 13-24. [CrossRef]

20. He, X.G. Method and System for Controlling a Multiple Input/Output Process with Minimum Latency Using a Pseudo Inverse Constant. U.S. Patent 5,892,679, 6 April 1999.

21. Zhe, Y.; Chao, Z.; Hong, Z.; Fei, F.; Shutao, C.; Xiaoming, C. Approximation of large time delay for mimo systems by using matrix pade-type. In Proceedings of the 2011 Second International Conference on Mechanic Automation and Control Engineering, Hohhot, China, 15-17 July 2011; pp. 108-111.

22. Sarma, K.L.N.; Chidambaram, M. Centralized pi/pid controllers for nonsquare systems with rhp zeros. J. Indian Inst. Sci. 2005, 85, 201-214.

23. Jin, Q.; Feng, H.; Qi, W. IMC-PID controller based on diagonal equivalent model for multivariable systems with multiple time delays. J. Chem. Eng. Jpn. 2013, 46, 209-218. [CrossRef]

(C) 2019 by the authors. Licensee MDPI, Basel, Switzerland. This article is an open access article distributed under the terms and conditions of the Creative Commons Attribution (CC BY) license (http://creativecommons.org/licenses/by/4.0/). 